\title{
Use of Acusto-Optic Tunable Filters for Multispectral and Hyperspectral Selection of Optical Images
}

\author{
M.O. Melnyk, R.M. Melnyk \\ Lviv research center of Institute for Space Research of \\ NAS and NSA of Ukraine, \\ Lviv, Ukraine \\ e-mail: melnykmo@isr.lviv.ua
}

\author{
H.I.Haskevych, D.M. Vynnyk \\ Lviv Physico-Mechanical Institute of NAS of Ukraine \\ e-mail: gas@ipm.lviv.ua
}

\begin{abstract}
The geometry of acousto-optic interaction in a $\mathrm{TeO}_{2}$ crystal suitable for design of tunable acousto-optic filters with enhanced spatial resolution is described. Some results of its evaluation are given, considering the applications in multispectral and hyperspectral image selection. The advantages of compact dimensions and low energy consumption are discussed regarding the use of such devices in onboard systems for small satellites.
\end{abstract}

Index Terms - acusto-optic tunable filter, multispectral and hyperspectral selection, Bragg's cell, spatial resolution.

\section{INTRODUCTION}

The spectral filtering in multispectral and hyperspectral optical systems used in remote sensing of Earth surface form space as well as in atmospheric research can be performed by different techniques. For instance, one can use prisms, diffraction gratings, various filters, etc. The choice of a particular selection method depends on the application of given multi- or hyperspectral apparatus, its design peculiarities and operation conditions. The filtering components mentioned above often are bulky, complex, expensive, and cosume quite high electric power.

An acusto-optic tunable filter (AOTF) is a relatively new selective element with electrically controllable spectral response. The operation of such devices is based on light diffraction on phase gratings induces by acoustic waves in birefringent crystals. A special geometry of acusto-optic anisotropic interaction is used, where the plane of this interaction coincides with the optical axis, and the acoustic wave vector has an angle of $\alpha \neq 0$ in respect to the normal to optical axis(1).

This filter can process the light beams with broad apertures. If a beam with a broad spectrum of $\lambda$ wavelengths strikes an AOTF, the $1^{\text {st }}$ Bragg's diffraction order collects a radiation in a narrow band of $\Delta \lambda$. The frequency of control signal allied to the piezo transducer sets the central wavelength of the $1^{\text {st }}$ Bragg's order, while its amplitude affects the intensity of diffracted light. The advantages of the AOTF include fast electronic retuning with the response down to microseconds, relative simplicity and high reliability thanks to the absence of movable parts [1,2].

The important peculiarity of light diffraction on acoustic waves is the dependence of phase synchronism on polarization of the incident light $[2,3]$. This phenomenon may allow one to

This work was carried out under support of research project... perform polarization-based image analysis. It has been shown $[4,5]$ that the studies on polarization of reflected light together with its spectral composition appears useful for detection of artificial objects on natural background. The acousto-optic filers operating with non-collinear and non-polarized light beams and splitting them into two ones with orthogonal polarization directions were studied in $[5,6]$. These studies dealt with AOTFs based on Bragg's cells made of $\mathrm{TeO}_{2}$ crystal with $\alpha \geq 100^{\circ}$. A drawback of such filters is a relatively low spatial resolution. The angular aperture of diffraction was $0.6^{\circ}$ [5]. Therefore it is desirable to find a geometry of anisotropic acousto-optic interaction in a crystal, with enhanced spatial resolution and ability to operate with non-collinear and nonpolarized light beam.

\section{EXPERIMENT AND RESULTS}

The present study deals with acousto-optic tunable filters with cells made of $\mathrm{TeO}_{2}$ crystals and featuring $\alpha<100^{\circ}(\alpha=$ $\left.60^{\circ}\right)$. The light wavelength for maximal diffraction intensity with this acousto-optic interaction geometry depends on acoustic wave frequency $f$ and light incidence angle $\Theta$ by the following expression [2]:

$$
\lambda=\frac{v}{f}\left[n_{i} \sin \Theta-\left(n_{0}^{2}-n_{i}^{2} \cos \Theta\right)^{1 / 2}\right]
$$

Here $n_{i}=n_{0} n_{e}\left[n_{e}^{2} \cos ^{2}(\Theta+\alpha)+n_{0}^{2} \sin ^{2}(\Theta+\alpha)\right]^{1 / 2} ; \quad n_{0}, \quad n_{e}-$ refraction indices for ordinary and extraordinary rays, $v-$ velocity of slow shear wave in the [110] plane of the $\mathrm{TeO}_{2}$ crystal. The $v$ velocity is defined as follows:

$$
v=\left[\left(\frac{c_{11}-c_{12}}{2} \cos ^{2} \alpha+c_{44} \sin ^{2} \alpha\right) / \rho\right]^{1 / 2} .
$$

Here $\rho$ and $c_{i j}-$ density and elasticity constants of $\mathrm{TeO}_{2}$ crystal.

The frequency dependences of diffracted light wavelength for $\lambda=0.63 \mu \mathrm{m}$ and different $\alpha$ values, according to (1), are shown in Fig. 1. The calculations were performed for $n_{0}=$ 2.26, $n_{e}=2,41, v=0.65 \cdot 10^{3} \mathrm{~m} / \mathrm{s}$ for $\alpha=60^{\circ}$ and $v=0.71 \cdot 10^{3}$ $\mathrm{m} / \mathrm{s}$ for $\alpha=9.50^{\circ}$ and $\alpha=100^{\circ}$, Bragg's angle is $\Theta_{\mathrm{B}}=130^{\circ}$ for 
all $\alpha$ values. It is evident that the operating frequencies for the same light wavelength range increase with $\alpha$. The operating frequency range and the central frequency for an acousto-optic cell based on the $\mathrm{TeO}_{2}$ crystal were determined accordingly to Fig. 1 to be $\Delta f=70.0 \mathrm{MHz}$ and $f_{0}=85.0 \mathrm{MHz}$, respectively.

An experimental prototype of an AOTF was created with a Bragg's cell based on $\mathrm{TeO}_{2}$ crystal, the geometry of the latter is shown in Fig. 2.

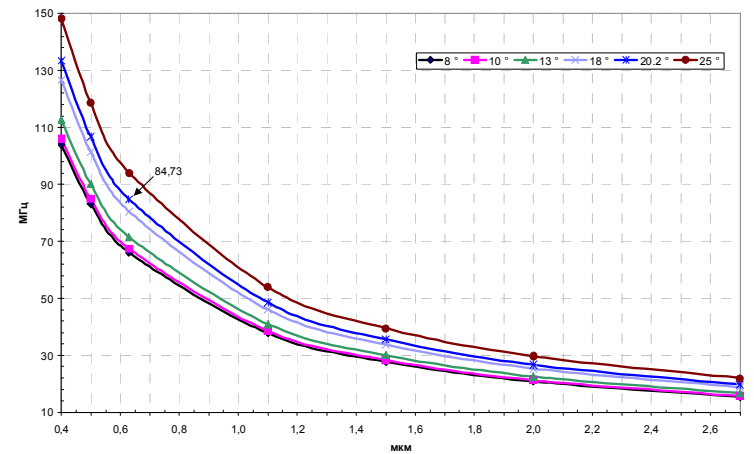

Fig.1. Calculated dependences for wavelengths of an AOTF based on $\mathrm{TeO} 2$ crystal on the frequency of electric control signal at visible and infrared spectral ranges, for given light incidence angles.

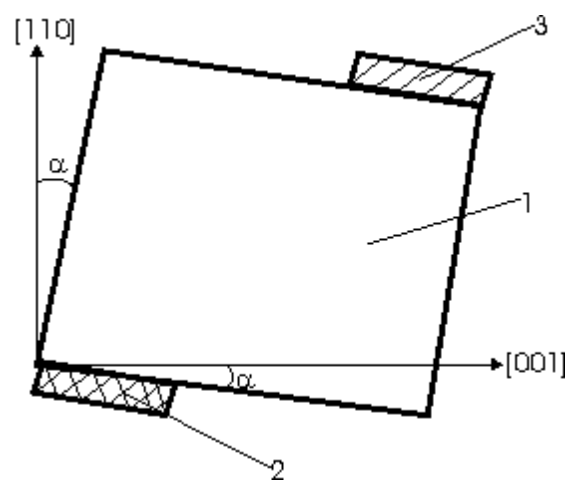

Fig.2. A Bragg's cell based on $\mathrm{TeO}_{2}$ crystal (1 - crystal, 2 - piezo transducer, 3 - absorber of acoustic waves).

The electric impedances for the output circuit of the HF generator and Bragg's cell were matched at a range of $50 \div$ $120 \mathrm{MHz}$. The diffraction efficiency for the control signal power of $\sim 0,3 \mathrm{~W}$ and at $\lambda=0.6$ мкм was $80 \%$. The performance of this AOTF was evaluated using a setup shown in Fig. 3

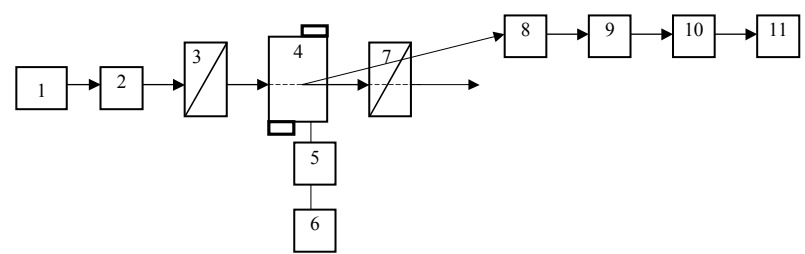

Fig.3. The experimental setup for evaluation of an AOTF prototype.

The light from a xenon lamp 1 was collimated by a lens 2 , while a necessary aperture was created by a diaphragm 3 . The beam after diaphragm was directed into the cell under test 4 , the piezo transducer 5 of the latter was driven by a HF oscillator 6 . The cell was installed on a turntable, thus making it possible to vary the angle of light incidence. The frequency of the HF oscillator was monitored by a counter 7 . The diffracted light was directed to a screen during setting the filter operation mode. The non-diffracted light was absorbed by an absorber 10. The polarization of the diffracted light was controlled by a polarizer 11 . The screen was replaced by a FD$24 \mathrm{~K}$ photodiode with a gap-like diaphragm for determining the parameters of the filter. The signal from this photodiode was fed to the PC via an amplifier and an ADC board. A selected spectrum of a light beam was observed at the plane of the Photosensor (diode), when a control signal of fixed frequency was applied to the piezo transducer. In such a case the setup shown in Fig. 3 operated as a spectrograph. If the frequency of the control HF signal was varied, the spectrum at the plane of Photosensor was scanned, so the setup was acting like a spectrometer.

The AOTF under test has demonstrated the following performance: The passband width was $\Delta \lambda_{1 / 2}=21.2 \mathrm{~nm}$ at $\lambda=$ $0.63 \mu \mathrm{m}$, for the given piezo transducer thickness of $l=1 \mathrm{~mm}$. The transit time of acoustic wave through the light beam aperture is a function of $h$, and in our case was

$$
t=\frac{h}{v}=\frac{3 \mathrm{~mm}}{0,65 \cdot 10^{3} \mathrm{~m} / \mathrm{s}}=4,6 \cdot 10^{-6} \mathrm{~s} .
$$

This AOTP at $\Theta_{\mathrm{B}}=130^{\circ}$ operated in a mode of nonpolarized light diffraction into $\pm 1^{\text {st }}$ order, with the orthogonal polarization direction for diffracted beams. The measured angular aperture of a non-collimated beam was 3/20 for this mode of operation.

\section{CONCLUSIONS}

The present studies have revealed that this geometry of acousto-optic interaction in $\mathrm{TeO}_{2}$ crystals can be used in design of AOTFs, which will be able to perform both spatial and spectral selection, with simultaneous polarization discrimination of the diffracted beams. The use of substantially lower frequencies of the control signal for the same operation wavelength range is an advantage of this geometry against competitors [5, 6]. This is a substantial factor for development of acousto-optic filters intended for operation with large beam apertures, because the attenuation of the acoustic waves at operating HF frequency range is much reduced. Moreover, the spatial resolution is substantially increased too. Therefore such AOTF can be successfully employed in stationary as well as spacecraft-borne systems for multispectral and hyperspectral selection of optical images.

\section{REFERENCES}

[1] Dixon R.W.// IEEE J.Quant.Electr.1967.V.3. P.85-93.

[2] Chang I.C.// Opt.Eng. 1981. Vol 20. №6. P. 824.

[3] Chang I.C.//Appl.Phys.Lett.1974. V.25. N.7. H.370-372.

[4] Gupta N., Denes L., Gottlieb M. et al // Appl.Opt. 2001. V.40. №36. P. 6626.

[5] Gupta N.//Proc.SPIE. 2008. Vol.6972. P.69720C 1-11/

[6] Glenar O., Hillman J., Saif B., Bergstralh J. // Appl.Opt. 1994, V.33. № 31. Р. 7412 